The Astrophysical Journal, 449:L105-L108, 1995 August 20

(C) 1995. The American Astronomical Society. All rights reserved. Printed in U.S.A.

\title{
DEEP OPTICAL GALAXY COUNTS WITH THE KECK TELESCOPE ${ }^{1}$
}

\author{
Ian Smail, ${ }^{2}$ David W. Hogg, ${ }^{3}$ Lin Yan, ${ }^{4}$ AND Judith G. Cohen ${ }^{4}$ \\ Received 1995 April 28; accepted 1995 June 15
}

\begin{abstract}
We present faint galaxy counts from deep VRI images obtained with the Keck Telescope. These images reach $R \sim 27$ in median seeing FWHM $\sim 0.5-0$. ". 6 , and we detect a integrated galaxy number density of $7 \times 10^{5} \mathrm{deg}^{-2}$, equivalent to $3 \times 10^{10}$ galaxies in the observable universe. In addition we present median galaxy colors as a function of magnitude; bluing trends are visible in all colors to $R \sim 24.5$. Fainter than $R \sim 24.5$, however, the typical $V-R$ color becomes redder again, $V-I$ remains constant, and $R-I$ becomes yet bluer. These trends are consistent with the $V R I$ count slopes, implying a decrease in the $V$ slope at the faintest levels, which our data support. Taking advantage of our good seeing we also present median half-light radii for faint galaxies; these show a steady decline at fainter magnitudes, leading to an intrinsic half-light radius of $\sim 0$ ". 2 for a typical $R \sim 26$ galaxy. Irrespective of the redshift distribution, the extremely high galaxy surface densities and their small intrinsic sizes are consistent with a scenario in which the majority of the very faint field population are dwarf galaxies or subgalactic units.
\end{abstract}

Subject headings: cosmology: observations — galaxies: evolution — galaxies: photometry

\section{INTRODUCTION}

The issue of the total number of galaxies in the visible universe is more than an academic curiosity; number counts as a function of apparent magnitude hold important clues to the formation and evolution of galaxies (see Koo \& Kron 1992 for a review). The study of very faint field galaxies using CCDs was pioneered by Tyson (1988) who found an integrated number density of $\sim 2 \times 10^{5} \mathrm{deg}^{-2}$, or $\sim 10^{10}$ galaxies over the whole sky at $B \sim 26$. Two more recent studies that are notable for their depth and resolution are those of the Hawaii group (Lilly, Cowie, \& Gardner 1991) and the Durham group (Metcalfe et al. 1991, 1995). The latter now reach galaxy surface densities in excess of $4 \times 10^{5} \mathrm{deg}^{-2}$ for a small field at a limiting magnitude of $B=27.5$. Equivalent surface densities are also being achieved in the near-infrared, $K$ (Gardner, Cowie, \& Wainscoat 1994; Cowie et al. 1995; Djorgovski et al. 1995).

Potentially the most interesting result from these studies, first observed by Lilly et al. (1991) and confirmed by Metcalfe et al. (1995) with deeper data, is the change in the slope, $\alpha$ (where $\log d N / d m \sim \alpha m$ ), of the deep $B$ counts. The differential galaxy number counts in the $B$ band show a decrease in slope from $\alpha_{B} \sim 0.5$ to $\alpha_{B} \sim 0.3$ at $B \sim 25$ (equivalent to $R \sim 24-24.5)$. In addition, Roche et al. (1993) claim that the amplitude of the two-point angular correlation function reaches a minimum around $B \sim 25$. Taken together these two observations have been interpreted by Metcalfe et al. (1995) as a signature of an increasing dominance of low-luminosity galaxies at $z \gtrsim 1$. These features, if confirmed and observed in other passbands, will constrain galaxy evolution models, although redshift information will be required before any strong conclusions can be drawn.

This Letter presents the first deep optical counts with the

\footnotetext{
${ }^{1}$ Based on observations obtained at the W. M. Keck Observatory, which is operated jointly by the California Institute of Technology and the University of California.

2 The Observatories of the Carnegie Institution of Washington, 813 Santa Barbara Street, Pasadena, CA 91101-1292.

3 Theoretical Astrophysics, Caltech 130-33, Pasadena, CA 91125.

${ }_{4}^{4}$ Palomar Observatory, Caltech 105-24, Pasadena, CA 91125.
}

$10 \mathrm{~m}$ Keck Telescope. These VRI observations are both deeper, at a given completeness limit, than previous published observations and more significantly were obtained in good seeing, FWHM 0.5-0."6, providing a wealth of information on the very faint field population.

\section{OBSERVATIONS AND ANALYSIS}

\subsection{Observations and Reduction}

The observations presented here were obtained by S. R. Kulkarni and J. G. Cohen to study the optical emission from two high galactic latitude pulsars. Thus for our purposes the fields provide randomly selected samples of the faint field population. These data were obtained on the night of 1994 August 8 using the Low Resolution Imaging Spectrograph (LRIS; Oke et al. 1995) on the $10 \mathrm{~m}$ Keck Telescope, Mauna Kea. The imaging mode of LRIS provides a $5.7 \times 7.3$ field onto a thinned Tek detector with 0.21 pixel $^{-1}$ sampling and $8.0 e^{-}$read noise. Conditions during this run were good, and the night was photometric; a log of the observations is given in Table 1. The raw frames, with individual exposure times between 0.5 and $1.5 \mathrm{ks}$, were debiased and then flat-fielded in a standard manner using dome flat fields. For each passband the dithered frames, with pointing offsets of $10^{\prime \prime}-20^{\prime \prime}$, were registered and combined using standard IRAF routines and a clipped-average algorithm to produce the final images. We reproduce a randomly chosen $1^{\prime} \times 1^{\prime}$ region from the $R$ frame of $1640+22$ in Figure 1 (Plate L11).

Photometric calibrations were derived from observations of Landolt (1992) standard stars at air masses very close to those of our science observations. We estimate the average errors in our absolute magnitude scales to be $\delta \lesssim 0.02$. Color differences between the standard stars and typical faint galaxies give systematic errors of a similar size. The estimated reddening is $E(B-V)=0.07$ (Stark et al. 1992) for both fields, and so we have applied zero-point corrections of $\delta V=0.16, \delta R=0.12$ and $\delta I=0.07$. We conservatively estimate our absolute calibrations to be good to $\lesssim 0.1$ mag. 
TABLE 1

LOG OF OBSERVATIONS

\begin{tabular}{|c|c|c|c|c|c|c|c|c|c|c|}
\hline Field & $\begin{array}{c}\alpha \\
(\mathrm{J} 2000)\end{array}$ & $\begin{array}{c}\delta \\
(\mathrm{J} 2000)\end{array}$ & Filter & $\begin{array}{l}T_{\exp } \\
(\mathrm{ks})\end{array}$ & $\begin{array}{c}N_{\gamma} \\
\left(10^{3} e^{-}\right)\end{array}$ & $\begin{array}{c}\mu(1 \sigma) \\
\left(\operatorname{mag} \operatorname{arcsec}^{-2}\right)\end{array}$ & $\begin{array}{l}m_{\lim } \\
(50 \%)\end{array}$ & $\begin{array}{c}N_{\lim } \\
(50 \%)\end{array}$ & FWHM & $\begin{array}{c}\text { Area } \\
\left(\operatorname{arcmin}^{2}\right)\end{array}$ \\
\hline $1640+22$. & $\begin{array}{c}16^{\mathrm{h}} 40^{\mathrm{m}} 18^{\prime \prime} 90 \\
l=41.1\end{array}$ & $\begin{array}{c}+22^{\circ} 24^{\prime} 19^{\prime \prime} 0 \\
b=38.3\end{array}$ & $\begin{array}{l}V \\
R \\
I\end{array}$ & $\begin{array}{l}1.5 \\
2.4 \\
2.0\end{array}$ & $\begin{array}{r}16.5 \\
44.3 \\
164.1\end{array}$ & $\begin{array}{l}28.31 \\
28.49 \\
27.06\end{array}$ & $\begin{array}{l}27.1 \\
26.9 \\
26.0\end{array}$ & $\begin{array}{l}4562 \\
5611 \\
4618\end{array}$ & $\begin{array}{c}0.78 \\
0.55 \\
0.53\end{array}$ & $\begin{array}{l}39.8 \\
40.2 \\
40.3\end{array}$ \\
\hline $2229+26 \ldots \ldots \ldots \ldots$ & $\begin{array}{c}22^{\mathrm{h}} 29^{\mathrm{m}} 50 . .89 \\
l=87.7\end{array}$ & $\begin{array}{c}+26^{\circ} 43^{\prime} 52^{\prime \prime} 8 \\
b=-26.3\end{array}$ & $\begin{array}{l}V \\
R \\
I\end{array}$ & $\begin{array}{l}0.9 \\
2.1 \\
1.0\end{array}$ & $\begin{array}{r}9.0 \\
41.3 \\
94.6\end{array}$ & $\begin{array}{l}28.33 \\
28.05 \\
26.34\end{array}$ & $\begin{array}{l}26.6 \\
26.9 \\
25.6\end{array}$ & $\begin{array}{l}1306 \\
5254 \\
3284\end{array}$ & $\begin{array}{l}0.87 \\
0.58 \\
0.58\end{array}$ & $\begin{array}{l}15.0 \\
40.8 \\
36.0\end{array}$ \\
\hline
\end{tabular}

Notes.-For each filter we quote the total exposure time, $T_{\text {exp }}$; the total number of sky photons detected per pixel, $N_{\gamma}$; the $1 \sigma$ surface brightness limit, $\mu(1 \sigma)$; the $50 \%$ completeness limit from our simulations, $m_{\text {lim }}$; the number of objects detected above this limit on the frame, $N_{\text {lim }}$; the FWHM of the seeing in arcseconds; and the total field area in square arcminutes.

\subsection{Analysis and Photometry}

To analyze these images we elected to use the SExtractor image analysis package (Bertin 1994). This package is a fast, image analysis program that can detect (using a standard isophotal limit and area cut algorithm), robustly deblend, and analyze sparse and moderately crowded galaxy fields. The program provides positions, shapes, image profiles, half-light radii, and isophotal and aperture magnitudes for all deblended objects detected on a frame. To detect objects we first smooth the frame with the point-spread function and then threshold it at a level of $2.5 \sigma$ of the sky noise in the raw frame and identify objects. This threshold provides a typical point source detection limit of $R \sim 27.2$ and appeared optimal in our incompleteness and noise object analyses.

To obtain total magnitudes for our galaxies we have followed the approach used by Djorgovski et al. (1995). For the fainter objects we use a fixed aperture (1..5 diameter or $\sim 2-3 \times$ FWHM) to measure magnitudes; these are then aperture corrected to a 5.6 diameter under the assumption that the objects have roughly stellar profiles, a correction of $\delta \sim-0.14$ at $R=26$. This assumption was tested by stacking many faint objects to make "average" faint galaxies with high enough signal-to-noise ratio for large-aperture photometry; we find that the average galaxies have aperture corrections close to those of the stars (as expected from their typical sizes; see below). For the brighter objects in the field, those with isophotal diameters larger than our adopted fixed aperture, we measure isophotal magnitudes above a surface brightness threshold of $0.9 \sigma$ of the sky noise (Table 1) and similarly correct to 5."6 diameter. The majority of the objects with magnitudes brighter than $R \sim 20.5$ are saturated in our frames; we do not present counts brighter than this limit. To measure colors for our objects, we smooth all the frames to the effective seeing of the worst frame (the $V$ data), before measuring photometry in 1..5 diameter apertures for all the objects selected from the $R$ catalogs.

Removal of stars from our object catalog is necessary at brighter magnitudes. With the good seeing experienced during these observations it is relatively straightforward to use image concentration to identify the stellar locus to a depth of $R=24.5$. Comparison of the colors of these candidate stars to those in Landolt (1992) confirms their identification and supports our estimates of the errors on our absolute color calibrations of $\lesssim 0.1 \mathrm{mag}$. At $R=24.5$ the stellar fraction is $\lesssim 6 \%$ and falling, so we do not apply any correction for stellar contamination fainter than $R=25$.

\subsection{Completeness Modeling}

Nondetection, failure to detect an object that is really there; false detection, detection of nonreal, noise objects; and magnitude errors, incorrect measurement of an object's flux, all plague faint galaxy counts. These problems become progressively worse at fainter magnitudes, with their relative importance depending upon the slope of the counts (because magnitude errors "scatter" plentiful faint objects into brighter magnitude bins; Eddington bias) and the details of the data reduction procedures.

We assess nondetection and magnitude errors simultaneously with a Monte Carlo simulation that involves adding artificial galaxies to the data and reapplying the detection algorithm. In order to simulate both the mean properties and morphological diversity of faint galaxies we create artificial objects by extracting galaxy images at magnitude levels for which errors are negligible and dimming them by $2.5 \mathrm{mag}$, at a constant angular size. It should be noted that if the scale sizes of galaxies decrease at fainter magnitudes (see $\S 3$ ), then this procedure will tend to overestimate our incompleteness. Extensive simulations $\left(>10^{4}\right.$ objects per frame, dropped in one at a time) were used to create a large matrix $P_{i j}$, each element of which is the probability that a galaxy with magnitude $m_{j}$ is detected with a measured magnitude $m_{i}$. This matrix contains all of the completeness and magnitude error information. On the assumption that the counts do not turn over quickly (i.e., $\alpha$ does not change rapidly with magnitude near the limit of the data), $P_{i j}$ can be projected into a total detection rate $r_{i}$, the ratio of number observed to true number at magnitude $m_{i}$. The true number of galaxies in bin $i$ can then be estimated by dividing the observed number $N_{i}$ by the detection rate $r_{i}$.

To correct for false detections we have created noise frames identical to our observations and geometrically remapped, combined, and analyzed them in the same manner as the observations. We subtract the number counts of noise objects found in these simulated frames from our observed counts. This correction is a few percent or less, even in our faintest magnitude bins.

The faintest magnitude at which the counts can be reasonably completeness-corrected is subjective; we truncate our counts at or before the $50 \%$ completeness level, beyond which the Poisson errors, or any other error estimates, lose their meaning. 


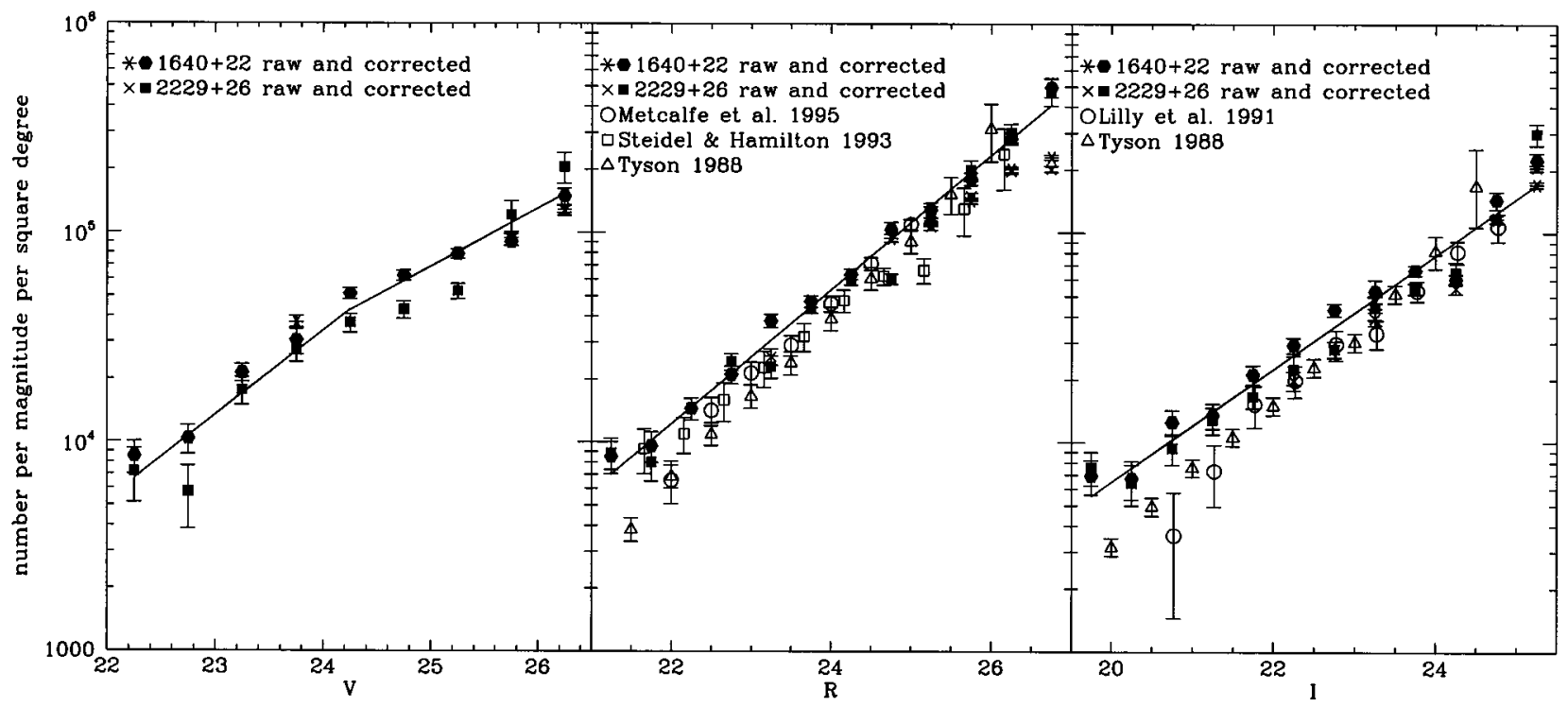

FIG. 2.-Plots of differential galaxy counts as a function of magnitude in the VRI passbands in the two fields. The raw and corrected counts are represented with open and filled symbols, respectively; circles for field $1640+22$, and squares for $2229+26$. The error bars are Poisson plus (on the filled points) an estimated uncertainty in the completeness correction. Least-squares linear fits are shown with solid lines (there are two fits to the $V$ counts split at $V=24.25$ ). Where applicable, the work of other authors is shown with open symbols; these are truncated at the same completeness limit as our data. The following corrections have been applied to transform the different passbands onto our system: Lilly et al. (1991) $I=I_{\mathrm{AB}}-0.48$ and Steidel \& Hamilton (1993) $R=\mathscr{R}_{\mathrm{AB}}+0.16$.

\section{RESULTS AND DISCUSSION}

We plot in Figure 2 the raw and corrected differential galaxy number counts from our two fields. We measure slopes from the combined field counts of $\alpha_{V}=0.404 \pm 0.015$ for $V=22$ 24.25 , flattening to $\alpha_{V}=0.28 \pm 0.05$ at fainter magnitudes, $\alpha_{R}=0.321 \pm 0.001$ for $R=21-27$ and $\alpha_{I}=0.271 \pm 0.009$ for $I=19.5-25.5$. We see no obvious variation in the $R$ and $I$ slopes. The evidence for the break in $V$ count slope at $V \sim 24.5$ is certainly not pronounced, but it is stronger in the deeper, wider area $1640+22$ data, and as we show below, it is also seen in the median galaxy colors. The integrated, corrected number counts are $2.9 \times 10^{5} \mathrm{deg}^{-2}$ to $V=26.5,7.3 \times 10^{5}$ to $R=27$, and $4.1 \times 10^{5}$ to $I=25.5$. We also plot on Figure 2 counts by other workers. The discrepancies in the count slopes at bright magnitudes may arise from other groups choosing fields devoid of bright galaxies; this biases them toward underdense regions producing a systematic undercount of galaxies at bright and intermediate magnitudes and thus a steeper slope to the counts. At faint limits we find reasonable agreement between the slopes of the various data sets, with residual differences in normalization consistent with the different photometric corrections applied by different workers and our quoted photometric errors.

The color distributions for our faint galaxy sample are shown in Figure 3. All show a bluing trend with fainter magnitude until $R \sim 24-24.5$, where a typical galaxy has a flat spectral energy distribution in $\nu f_{v}$ (corresponding to $V-R \sim 0.5$ and $R-I \sim 0.5)$. Fainter than $R \sim 24-24.5$, the typical $V-R$ colors turn around and become redder, while the $V-I$ colors flatten out and the $R-I$ continue to become bluer (see Steidel \& Hamilton 1993). If we are observing the same population in all three bands, then these trends in median color should be reflected in different count slopes in $V R I$. At $R \lesssim 24$ the bluing trends indicate $\alpha_{V}>\alpha_{R}>\alpha_{I}$ as observed. After the turnaround, the colors imply a decrease in $\alpha_{V}$ from steeper than $I$ at the bright end, to matching the $I$ slope at the faint end. This change in $V$-band slope is indeed seen in the counts, although its significance is not high.

The good seeing of our observations allows us to study the angular sizes of the faint galaxy population. We plot in Figure 4 the observed half-light radii, $r_{\mathrm{hl}}$, measured from the $R$-band images. A gradual, near-linear, decline is seen in the

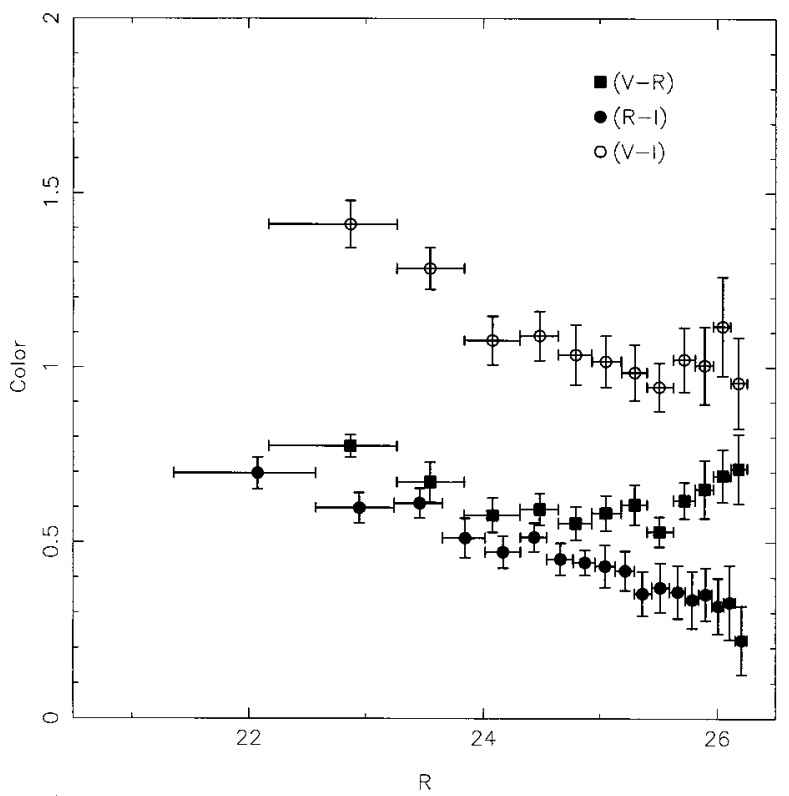

FIG. 3.-Typical colors of the faint galaxy population as a function of $R$ magnitude in the two fields. The points are medians of samples of 401 galaxies in each bin. The horizontal error bars show the extent of the magnitude bin, while the vertical error bars are $95 \%$ confidence limits calculated using bootstrap resampling of the data; nondetections are included. One sigma limits within our photometry aperture lie outside the region of the color planes plotted. Note the turnaround in the $V-R$ colors at $R \sim 24.0-24.5$ and the flattening of the $V-I$ colors at the same magnitude. 


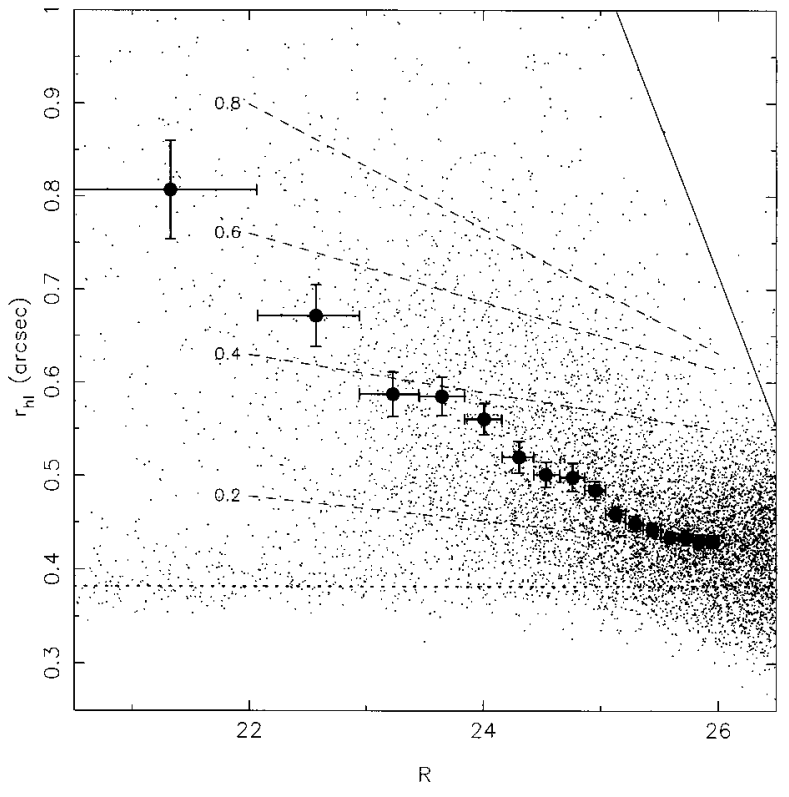

FIG. 4.- Half-light radii as a function of apparent $R$ magnitude for all the objects in our two fields. The points are medians of samples of 401 galaxies with error bars defined in a similar manner to Fig. 3 . The seeing difference between the fields has been removed by shifting the $2229+26$ points. The dotted line marks the locus of stars; these are excluded from the medians. Each of the dashed lines is a linear fit to the variation of apparent half-light radius with source magnitude for an exponential disk with a fixed, intrinsic half-light radius, labeled in arcseconds. The solid line shows the detection limit as a function of half-light radius for an exponential disk. For the most extended sources $\left(r_{h l} \gtrsim\right.$ 0 ".6) a bias is visible, such that the source sizes are progressively underestimated at fainter magnitudes. This bias arises from a combination of incompleteness beyond $R \sim 25$ and the use of inappropriate aperture corrections; the corrections applied are calculated for "typical," hence compact, faint galaxies. Nevertheless, it is apparent that the median source size in the field shrinks more rapidly than expected for a population with a fixed intrinsic angular size. By $R \sim 25.5-26$ the half-light radii are asymptotically approaching the stellar locus and indicate a very small intrinsic size for these faint galaxies, $r_{\mathrm{hl}} \sim 0$ ". 2 .

median size of faint galaxies to $R \sim 25.5-26$, at which point the median size is only just distinguishable from the stellar locus. Representing the faint field population as exponential disks leads to an estimate of the intrinsic half-light radius of a typical $R=25.5-26$ galaxy of $r_{\mathrm{hl}} \sim 0$ ".2. This angular size corresponds to $<2.9 h_{50}^{-1} \mathrm{kpc}$ at any redshift in any world model, so it is clear that the typical objects we are detecting are either intrinsically smaller or produce a larger fraction of their emission in their nuclear regions than local bright galaxies. Splitting the sample in two in each magnitude bin on the basis of $V-I$ color shows no statistical difference between the sizes of the blue and red galaxies.

In conclusion, we have presented deep galaxy counts in VRI passbands. We find a decrease in slope in our bluest passband, $V$, at the faint end similar to that seen in the $B$-band by Metcalfe et al. (1995) at an equivalent apparent magnitude. Beyond $R \sim 24$ it appears that galaxy counts approach log $d N / d m \sim 0.3 m$ in all bands, shallow enough to remove the threat of a divergence in the integrated night sky brightness. If we are not seeing to very high redshift, either because the universe is Einstein-de Sitter and we are running out of volume, or because there are few luminous, high-redshift field galaxies, then the common faint end slope in the galaxy counts is a measure of the shape of the galaxy (or possibly pregalactic fragment, in view of the small angular size of the faintest objects) luminosity function at the faint end. In these scenarios, the slope of 0.3 implies a faint end luminosity function $\Phi(L) \sim L^{-1.75}$ at large look-back times, in contrast to $L^{-1}$ observed locally (e.g., Loveday et al. 1992). The faint counts may thus confirm, at higher redshift, the steepening of the faint end slope of the luminosity function suggested by analyses of spectroscopic samples at brighter magnitudes (Eales 1993; Ellis et al. 1995). Interestingly, the rollover at $V \sim 24.5$ is within reach of the new generation of large telescopes, and therefore we may hope for direct spectroscopic observations of galaxies in this intriguing magnitude range.

First, we thank Shri Kulkarni for his great generosity in allowing us to use these data. We acknowledge useful discussions and encouragement from Rebecca Bernstein, Roger Blandford, and Nigel Metcalfe. We also thank the referee, Richard Kron, for many helpful comments. Support via a NATO Advanced Fellowship and a Carnegie Fellowship (I. R. S.) and an NSF Graduate Fellowship (D. W. H.) is gratefully acknowledged. Finally, it is a pleasure to thank the W. M. Keck Foundation and its president, Howard B. Keck, for the generous grant that made the W. M. Keck Observatory possible.

\section{REFERENCES}

Bertin, E. 1994, SExtractor Manual (Paris: IAP)

Cowie, L. L., Gardner, J. P., Hu, E. M., Songaila, A., Hodapp, K. W., \& Wainscoat, R. J. 1995, ApJ, 434, 114

Djorgovski, S., et al. 1995, ApJ, 438, L13

Eales, S. 1993, ApJ, 404, 51

Ellis, R. S., Colless, M. M., Broadhurst, T. J., Heyl, J. S., \& Glazebrook, K 1995, MNRAS, submitted

Gardner, J., Cowie, L. L., \& Wainscoat, R. J. 1993, ApJ, 415, L9

Koo, D. C., \& Kron, R. G. 1992, ARA\&A, 30, 613

Landolt, A. U. 1992, AJ, 104, 340

Lilly, S. J., Cowie, L. L., \& Gardner, J. P. 1991, ApJ, 369, 79
Loveday, J., Peterson, B. A., Efstathiou, G., \& Maddox, S. J. 1992, ApJ, 390, 338

Metcalfe, N., Shanks, T., Fong, R., \& Jones, L. R. 1991, MNRAS, 249, 498 Metcalfe, N., Shanks, T., Fong, R., \& Roche, N. 1995, MNRAS, 273, 257

Oke, J. B., et al. 1995, PASP, in press

Roche, N., Shanks, T., Metcalfe, N., \& Fong, R. 1993, MNRAS, 263, 368

Stark, A. A., Gammie, C. F., Wilson, R. W., Bally, J., Linke, R. A., Heiles, C., \& Hurwitz, M. 1992, ApJS, 79, 77

Steidel, C. C., \& Hamilton, D. 1993, AJ, 105, 2017

Tyson, J. A. 1988, AJ, 96, 
PLATE L11

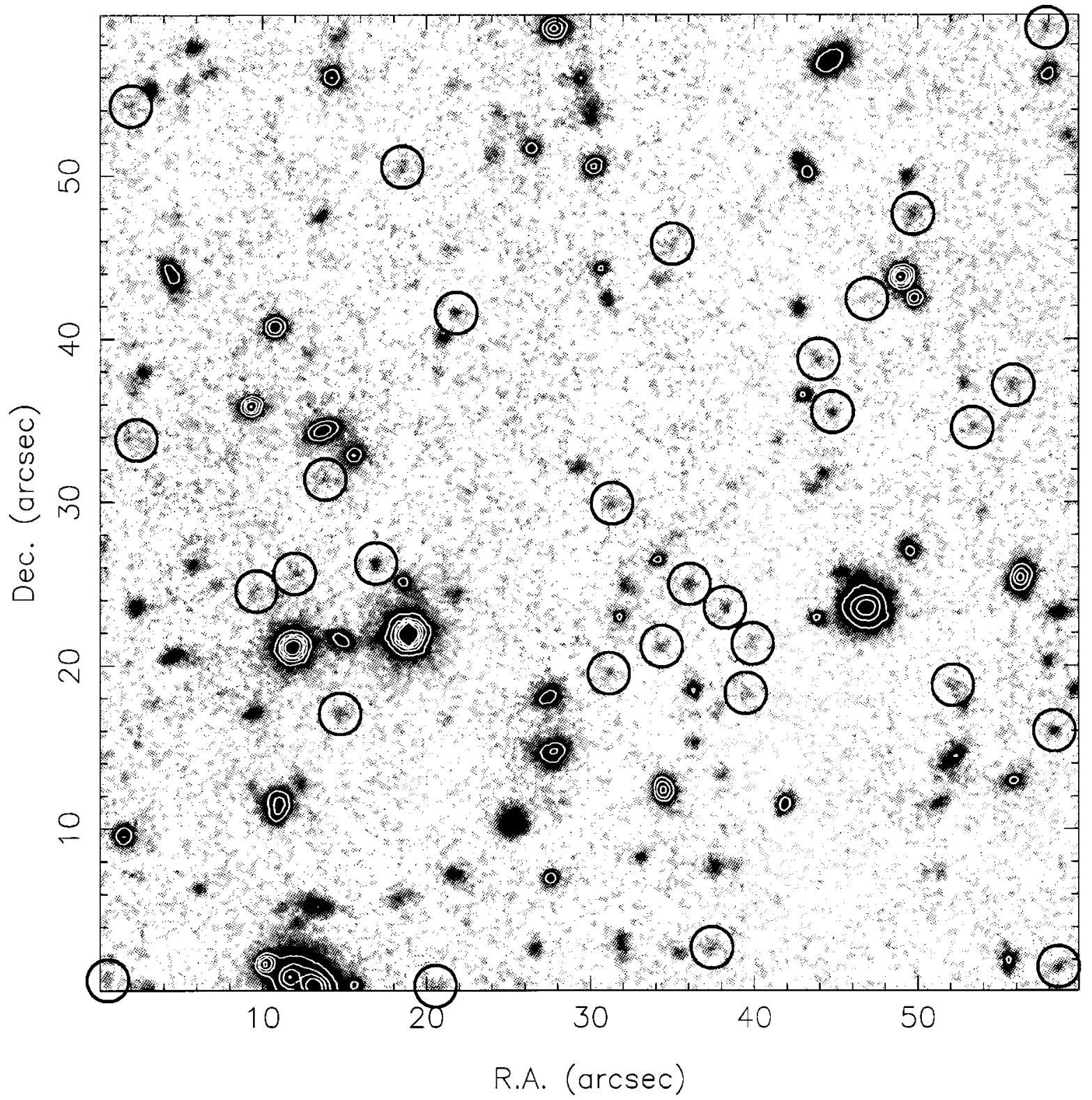

FIg. 1.-A randomly selected $1^{\prime} \times 1^{\prime}$ field from the $R$ image of $1640+22$. Objects with magnitudes in the range $R=26.0-26.5$ are marked. Notice the minimal crowding in the frame.

SmaIl et al. (see 449, L105) 